\title{
Isolation of Mycobacterium arupense from pleural effusion: culprit or not?
}

\author{
Xian Zhou ${ }^{1 \dagger}$, Qiaoling Ruan ${ }^{1 \dagger}$, Weimin Jiang ${ }^{1}$, Xinyu Wang ${ }^{1}$, Yuan Jiang ${ }^{3}$, Shenglei Yu ${ }^{1}, Y u X^{2}$, Jing Li ${ }^{3}$, \\ Yangyi Zhang ${ }^{3}$, Wenhong Zhang ${ }^{1}$ and Yuekai Hu ${ }^{1 *}$
}

\begin{abstract}
Background: Mycobacterium arupense, first identified in 2006, is a slow-growing nontuberculous mycobacterium (NTM) and an emerging cause of tenosynovitis, potentially associated with immunosuppression. However, unlike the diagnostic value of its isolation from osteoarticular specimens, the significance of detecting $M$. arupense in respiratory specimens is not yet clear.

Case presentation: To our knowledge, we, for the first time, described the identification of $M$. arupense from the pleural effusion of an immunocompetent patient, who presented with fever and chylothorax. The symptoms resolved with doxycycline treatment for 45 days and a low-fat, high-protein diet. Follow-up at 14 months showed no relapse.

Conclusions: Because the patient fully recovered without combined anti-NTM treatment, we did not consider M. arupense the etiological cause in this case. This indicates that $M$. arupense detected in pleural effusion is not necessarily a causative agent and careful interpretation is needed in terms of its clinical relevance.
\end{abstract}

Keywords: Mycobacterium arupense, Nontuberculous mycobacterium, Pleural effusion, Chylothorax

\section{Background}

Mycobacterium arupense, first identified in 2006, is a slowgrowing nontuberculous mycobacterium (NTM) belonging to $M$. terrae complex [1]. Although it is widespread in the environment, $M$. arupense is considered to cause NTM infection rarely, most commonly affecting patients with immunodeficiency or history of injury [2]. Here, we describe the identification of this species in the pleural effusion of an immunocompetent patient.

\section{Case presentation}

A previously healthy, 50-year-old farmer was admitted because of fever and generalized edema. Two weeks prior to hospitalization, fever (up to $39.8^{\circ} \mathrm{C}$ ), chills, and sore throat developed. Physical examination at a local hospital did not reveal significant findings, but only an oral ulcer. A week later, pharyngalgia worsened and shortness of breath developed. Laryngoscopy showed

\footnotetext{
* Correspondence: 13564579738@163.com

${ }^{\dagger}$ Equal contributors

${ }^{1}$ Department of Infectious Diseases, Huashan Hospital, Fudan University, 12

M. Wulumuqi Road, Shanghai 200040, China

Full list of author information is available at the end of the article
}

severe edema of the epiglottis and arytenoid cartilage, and chest computed tomography revealed bilateral pleural effusion (Fig. 1a). Diagnosed as acute laryngitis, $10 \mathrm{mg}$ dexamethasone was administered intravenously daily for 4 days. The patient's edema of the epiglottis and fever improved, but the edema extended to the whole body. After discontinuation of dexamethasone, the patient experienced a fever of $38{ }^{\circ} \mathrm{C}$. Therefore, he was transferred to our hospital.

On physical examination, the patient showed systemic edema, most severe in the scrotum and both the lower extremities. The white blood cell (WBC) count was $7.03 \times 10^{9} / \mathrm{L}$, with $75.2 \%$ neutrophils. Serum albumin, pro-BNP, and C-reactive protein levels were $27.9 \mathrm{~g} / \mathrm{L}, 55.7 \mathrm{pg} / \mathrm{mL}$, and $6.3 \mathrm{mg} / \mathrm{L}$, respectively. The patient had no diabetes mellitus. The serum immunoglobulin levels were within normal ranges. HIV was negative with CD4 counts of $531 / \mathrm{mm}^{3}$. Pleural fluid (Fig. 1b) analysis showed: total protein, $35.5 \mathrm{~g} / \mathrm{L}$; lactate dehydrogenase, $126 \mathrm{U} / \mathrm{L}$; red blood cell, $120 \times 10^{6} / \mathrm{L}$; WBC, $160 \times 10^{6} / \mathrm{L}$ with $2 \%$ neutrophils, $35 \%$ lymphocytes, and $63 \%$ macrophages. The fever was persistent even after the empirical meropenem treatment. Repeated blood

(c) The Author(s). 2018 Open Access This article is distributed under the terms of the Creative Commons Attribution 4.0 International License (http://creativecommons.org/licenses/by/4.0/), which permits unrestricted use, distribution, and 


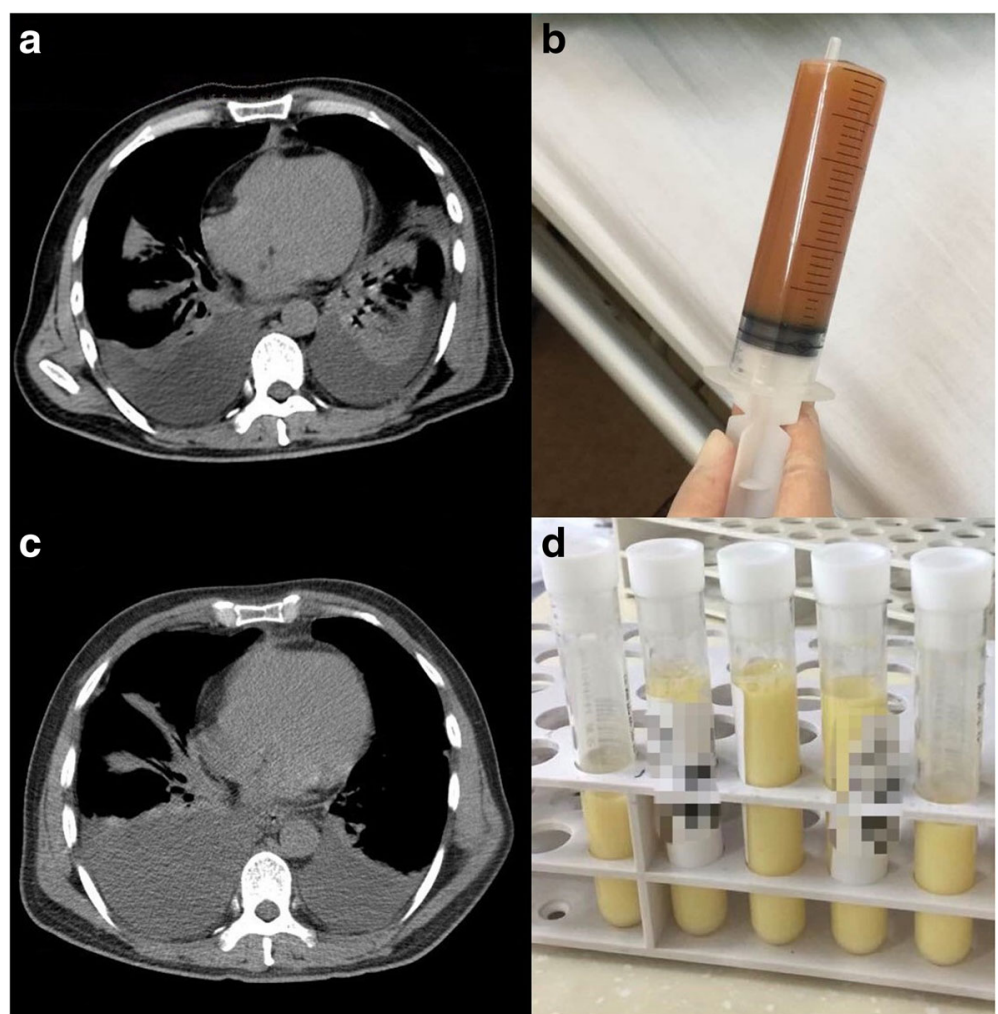

Fig. 1 Pleural effusion of a 50-year-old male farmer. a Computed tomography revealed bilateral pleural effusion about a week after the onset of symptom. b Pleural effusion from the first thoracentesis. c Computed tomography showed that pleural effusion continued. $\mathbf{d}$ Pleural effusion from the second thoracentesis revealed chylothorax

cultures were negative for bacteria and fungi. As the patient reported close contact with cats and dogs, doxycycline was added in the treatment and the patient's temperature returned to normal immediately. However, the edema and bilateral pleural effusion continued despite the diuretics and albumin supportive treatment. The second thoracentesis confirmed chylothorax (Fig. 1c-d), with triglyceride levels at $1389 \mathrm{mg} / \mathrm{dL}$. Malignant cells were not found in the pleural effusion. Thick blood film smears for microfilariae and vascular ultrasound for thrombus showed negative results. Therefore, we decreased the patient's intravenous fluid supplement and the symptoms were relieved with a low-fat, high-protein diet.

Mycobacterium species from the first pleural effusion specimen were cultured successfully on the LöwensteinJensen medium after 23 days. The culture smear was positive for acid-fast bacilli. Following inoculation to MGIT 960 liquid system and the solid LöwensteinJensen medium were both positive for mycobacteria. The partial 16S rRNA gene sequence of the isolate (482 bp) was $99.17 \%$ similar to that of the $M$. arupense strain AR30097. The susceptibility testing of the isolates for capreomycin and moxifloxacin yielded minimum inhibitory concentrations of $64 \mu \mathrm{g} / \mathrm{mL}$ and $>16 \mu \mathrm{g} / \mathrm{mL}$, respectively. By the time $M$. arupense was confirmed, the patient recovered and refused the proposal of anti-NTM treatment. Follow-up at 3 months after discharge showed no pleural effusion, and follow-up at 14 months showed no relapse.

\section{Discussion and conclusions}

Isolation of $M$. arupense has been reported from at least 118 clinical samples [2]. These strains were detected mainly in two types of specimens: 26 in tissue specimens and 92 in respiratory specimens (Additional file 1: Table S1). Only one positive blood culture report was obtained from an acquired immune deficiency syndrome (AIDS) patient who was diagnosed with disseminated $M$. arupense infection [3]. Ten out of 26 strains from tissue specimens were reported in a 2016 study by Vasireddy et al. [4]. They identified $M$. arupense by sequencing the 26 clinical isolates of tenosynovitis or osteomyelitis previously diagnosed as $M$. terrae and $M$. nonchromogenicum from 1984 to 2014 in the U.S. Patients with $M$. arupense osteoarticular infection usually had a history of an injury or corticosteroid use. Although there is no standard well-established treatment for $M$. arupense infection, these patients showed favorable prognosis after surgery and 614 months of combined antimicrobial treatment. 
Unlike the diagnostic value of its isolation in osteoarticular specimens, the significance of detecting $M$. arupense in respiratory specimens is not yet clear. Since the first $M$. arupense pulmonary infection in a patient with kidney neoplasm reported in 2010 [5], M. arupense has only been reported as a pathogen causing pulmonary infection in 2 other patients, both with AIDS [3,6] (Additional file 1: Table S1). Fifty-three of $92 \mathrm{M}$. arupense isolates from respiratory specimens were reported in a study retrospectively reviewing 53 cancer patients with positive cultures of M. arupense from 2007 to 2014 [7]. The outcomes of 13 treated patients and 40 untreated ones did not differ significantly. These results suggest that $M$. arupense can cause pulmonary infection in immunocompromised patients, but is usually a "bystander" in respiratory infections.

This is the first case report, to our knowledge, on the isolation of $M$. arupense from pleural effusion. There were isolations reported previously [1, 7], but with minimal clinical information. Although the possibility of contamination is low, we did not consider $M$. arupense as an etiological cause in our case for the following three reasons. First, NTM that was isolated from the pleural effusion should not be considered etiologic unless there is evidence of NTM infection in other tissues [8]. Second, the patient recovered with doxycycline treatment for 45 days. Although we did not perform a drug susceptibility test to doxycycline of the isolate due to limited resources, drug susceptibility testing of 40 strains of $M$. arupense reported by Beam et al. found that only 5 out of $23(21.7 \%)$ isolates were susceptible to doxycycline [9]. There is no report of doxycycline mono treatment for NTM cases. Lastly, NTM related chylothorax has been reported in only one AIDS patient [10], but not in immunocompetent patients. Although these observations are based on a single patient and lack a control group or other studies for comparison, this case indicates that $M$. arupense found in pleural effusion is not necessarily a causative agent and careful interpretation is required to pinpoint its clinical relevance.

\section{Additional file}

Additional file 1: Table S1. Summary of pulmonary isolates of $M$. arupense. A table of cases with pulmonary isolates of $M$. arupense over the years with clinical history. (DOCX $20 \mathrm{~kb}$ )

\section{Abbreviations}

AIDS: Acquired immune deficiency syndrome; NTM: Nontuberculous mycobacterium; WBC: White blood cell

\section{Availability of data and materials}

All data generated or analyzed during this study are included in this published article and its supplementary information files.

\section{Authors' contributions}

Conception or design of the work: XZ, QR, WZ and YH. Data collection and patient care: $X Z, Q R, X W, Y J, S Y, Y X, J L, Y Z, Y H$. Data analysis and interpretation:
$X Z, Q R, W J, X W, Y J, S Y, J L, Y Z, W Z, Y H$. Drafting the article: XZ and QR. Critical revision of the article: $W Z$ and $Y H$. Final approval of the version to be published: $X Z, Q R, W J, X W, Y J, S Y, Y X, J L, Y Z, W Z$ and $Y H$.

Ethics approval and consent to participate

The study adhered to CARE guidelines. We identified this patient during routine clinical practice and he consented to give venous blood samples after elaborate information. Involvement of the ethical committee of the Huashan Hospital of Fudan University was considered unnecessary, since the project was not based on a study protocol.

\section{Consent for publication}

Written informed consent was obtained from the patient for publication of this case report and all accompanying images. A copy of the written consent is available for review by the editor of this journal.

\section{Competing interests}

The authors declare that they have no competing interests.

\section{Publisher's Note}

Springer Nature remains neutral with regard to jurisdictional claims in published maps and institutional affiliations.

\section{Author details}

'Department of Infectious Diseases, Huashan Hospital, Fudan University, 12 M. Wulumuqi Road, Shanghai 200040, China. ${ }^{2}$ Department of Laboratory Medicine, Huashan Hospital, Fudan University, Shanghai, China. ${ }^{3}$ Shanghai Municipal Center for Disease Control and Prevention, Shanghai, China.

Received: 9 February 2018 Accepted: 7 May 2018

Published online: 15 May 2018

\section{References}

1. Cloud JL, Meyer JJ, Pounder Jl, Jost KC Jr, Sweeney A, Carroll KC, Woods GL. Mycobacterium arupense sp. nov., a non-chromogenic bacterium isolated from clinical specimens. Int J Syst Evol Micr. 2006;56(6):1413-8.

2. Abudaff NN, Beam E. Mycobacterium arupense: a review article on an emerging potential pathogen in the Mycobacterium terrae complex. Journal of Clinical Tuberculosis and Other Mycobacterial Diseases. 2017;10:1-5.

3. Heidarieh P, Hashemi-Shahraki A, Khosravi AD, Zaker-Boustanabad S, Shojaei $\mathrm{H}$, Feizabadi MM. Mycobacterium arupense infection in HIV-infected patients from Iran. Int J STD AIDS. 2013;24(6):485-7.

4. Vasireddy R, Vasireddy S, Brown-Elliott BA, Wengenack NL, Eke UA, Benwill $J$, Turenne C, Wallace RJ. Mycobacterium arupense, Mycobacterium heraklionense, and a newly proposed species,"Mycobacterium virginiense" sp. nov., but not Mycobacterium nonchromogenicum, as species of the Mycobacterium terrae complex causing tenosynovitis and osteomyelitis. J Clin Microbiol. 2016;54(5):1340-51.

5. Neonakis IK, Gitti Z, Kontos F, Baritaki S, Petinaki E, Baritaki M, Liakou V, Zerva L, Spandidos DA. Mycobacterium arupense pulmonary infection: antibiotic resistance and restriction fragment length polymorphism analysis. INDIAN J MED MICROBI. 2010;28(2):173.

6. Varghese B, Enani M, Shoukri M, AlThawadi S, AlJohani S, Al-Hajoj S. Emergence of rare species of nontuberculous mycobacteria as potential pathogens in Saudi Arabian clinical setting. PLOS NEGLECT TROP D. 2017;11(1):e5288.

7. Al Hamal Z, Jordan M, Hachem RY, Alawami HM, Alburki AM, Yousif A, Deshmukh P, Jiang Y, Chaftari A, Raad II. Mycobacterium arupense in Cancer patients. Medicine. 2016;95(14):e2691.

8. Gribetz AR, Damsker B, Marchevsky A, Bottone EJ. Nontuberculous mycobacteria in pleural fluid: assessment of clinical significance. Chest. 1985:87(4):495-8.

9. Beam E, Vasoo S, Simner PJ, Rizzo M, Mason EL, Walker RC, Deml SM, Brown-Elliott BA, Wallace RJ, Wengenack NL, et al. Mycobacterium arupense flexor tenosynovitis: case report and review of antimicrobial susceptibility profiles for 40 clinical isolates. J Clin Microbiol. 2014;52(7):2706-8.

10. Tanaka T, Saito N, Takaki M, Furumoto A, Morimoto K, Ariyoshi K. Refractory chylothorax in HIV/AIDS-related disseminated mycobacterial infection. Thorax. 2016;71(10):960-1. 